\title{
DETECTION OF TOXOPLASMOSIS IN ENVIRONMENTAL SAMPLES AT A WET MARKET OF A CAPITAL CITY CENTRE
}

\begin{abstract}
Amal R. Nimir, Tang Cher Linn
Universiti Kebangsaan Malaysia, Jalan Raja Muda Abdul Aziz, 50300, Kuala Lumpur, Malaysia

Summary: The local Chow Kit market is the largest wet market in the city of Kuala Lumpur. It is very close to the biggest government hospital in the city centre. However, the level of cleanliness in this area is always questionable and a matter of concern. The aim of this study was to identify the prevalence of $T$. gondii oocyst in water samples used by hawkers in that market and tissue cysts in rats' brains captured from the same area.

Water samples were taken to the parasitology laboratory at the National Universtiy of MalaysiaUniversity and a sugar flotation concentration method was used. Supernatant microscopical examination was then performed. A total of 752 slides were screened for the presence of $T$. gondii oocyst. A hundred rats wandering in the same area were also captured by the hawkers using mousetraps. After each animal was sacrificed, and an electric microtome was used to cut out serial sections $5 \mu$ thick from the rat brains. The de-waxed tissue sections were stained by the progressive Haematoxylin and Eosin (H\&E) stain for microscopical examination.

A total of 1000 slides were screened under a light microscope to detect the presence of $T$. gondii brain cysts. All the water samples were found to be negative for $T$. gondii oocyst. Out of the 100 rats captured, three rats were found to possess T. gondii cysts in their brains.

Water samples reflect minimal or no solid food contamination, while the $3 \%$ of positive brain cysts influence the researchers to broaden their investigations for future projects.
\end{abstract}

Key words: T. gondii; Transmission; Environmental samples; Intermediate host; Water contamination

\section{Introduction}

Toxoplasma gondii infections are widely prevalent in human beings and animals worldwide. Its definitive hosts are cats and other Felidae. People typically become infected by three principal routes of transmission: food borne, animal-to-human (zoonotic) and mother-to-child (congenital). Presently, there are no tests to identify the source of infection in an individual, and evidence is based exclusively on epidemiological surveys.

Toxoplasmosis is common and has a high prevalence rate among the Malaysian population. The majority of infections are mild, with most people being asymptomatic (chronic/ latent infection). From 1985 to present, the trend of toxoplasmosis prevalence has shown a similar figure of $20-30 \%$ $(8,9,16)$.

Toxoplasmosis, until recently, was not considered a water-borne zoonosis. Some results show an association with $T$. gondii in municipal waters and marine mammals, suggesting that the contamination of seawater with $T$. gondii may be more common than actually identified (3). In certain areas of Brazil, approximately $60 \%$ of 6-8-year-old children have antibodies to $T$. gondii linked to the ingestion of oocysts in a heavily contaminated environment with
T. gondii oocysts (1). It is believed that the domestic cat is essential for the maintenance of the transmission cycle, and added to this is the unhealthy habits of meat consumption $(6,10,12,15)$. Interestingly, the Atlanta outbreak was linked to nasopharyngeal ingestion of oocysts aerosolized in the riding stable where $T$. gondii infected cats were present (11).

T. gondii oocysts are extremely resistant to environmental influences and can sporulate and survive in soil or water at $4{ }^{\circ} \mathrm{C}$ for months. However, at any given time only $1 \%$ of cats shed oocysts (5). Therefore, for epidemiological surveys, detection of $T$. gondii oocysts in cat feces is impractical and less informative. To the best of our knowledge, there are no commercial reagents available to detect $T$. gondii oocysts in the environment.

The aim of this study was to identify the prevalence of $T$. gondii oocyst in water samples that are widely used by hawkers in a local market in Kuala Lumpur and in tissue cysts in rats' brains captured from the same area.

\section{Materials and methods}

The study was performed between August 2008 and March 2009. The study design is a prospective laboratory 
based study. Prior ethical approval from the National Universtiy of MalaysiaUniversity was given for the study.

There are 177 workers and 153 small shops in the Chow Kit market. Only 133 shops were included in the study (owners of the remaining shops refused to be enrolled in this study). The selection criteria planned to be according to hygienic state, but we found that all shops possess a poor hygienic state. Therefore, no specific selection criteria were established, and all shops selling vegetables and fruits were enrolled after getting the owners'consent.

Every evening, the researchers collected water that had been used for the whole day by the hawkers. The water samples collected were used by the vendors to wash vegetables, fruits, tableware and hands. Therefore, these samples of water served as a good indication for the presence of $T$. gondii oocyst in solid food in the local Chow Kit market. The used water was kept in buckets, and the researchers collected it daily. The total amount of water collected during the time of study was 77 liters. A total number of 135 drinking water samples were taken. Collected water samples were taken to the parasitology laboratory at the National University of Malaysia and a sugar flotation concentration method was used. This technique separates the oocyst from other debris. The principle of this technique is that the specific gravity (SG: 1.200) used should float the cysts without changing their specific gravity, which might cause swelling or shrinking. The supernatant layer was removed from the tube with a pipette and placed on two clean glass slides for microscopical examination. A permanent Trichrome stain technique was used to help identify the parasites. A total of 752 slides were screened for the presence of $T$. gondii oocyst. The slide examination was done by a technologist who has 30 year experience in identifying protozoa.

One hundred rats wandering in the same area (Chow Kit market) were also captured by the hawkers using mousetraps. Each morning, the researcher collected the rats and took them back to the Anatomy Department at the National University of Malaysia. Open ether anesthesia was used for 90 seconds on average. After each animal was sacrificed, the whole brain of each rat was removed carefully. The left and right brain hemispheres were divided carefully. For each hemisphere, fixation and paraffin-wax embedding was followed by electric microtome sectioning which cut serial sections $5 \mu$ in thickness. The de-waxed tissue sections were stained by the progressive Haematoxylin and Eosin (H\&E) stain for microscopical examination. There were a total of 1000 slides which were screened under light microscope to detect the presence of $T$. gondii tissue cyst.

\section{Results}

All the water samples were screened and found to be negative for $T$. gondii oocyst.

Out of the 100 rats captured, three rats were found to possess $T$. gondii tissue cysts in their brain. One of them had

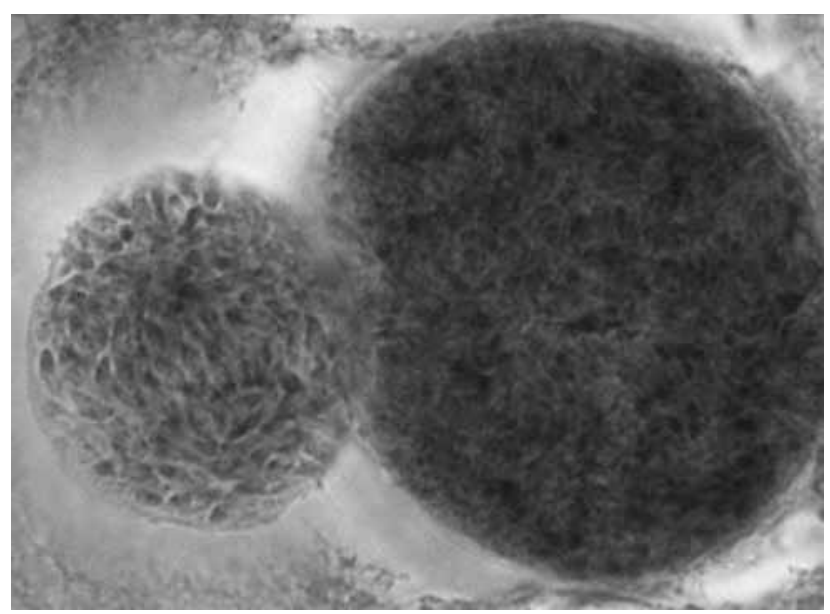

Fig. 1: Photograph showing two tissue cysts in the left hemisphere of rat number $1(\times 1000)$

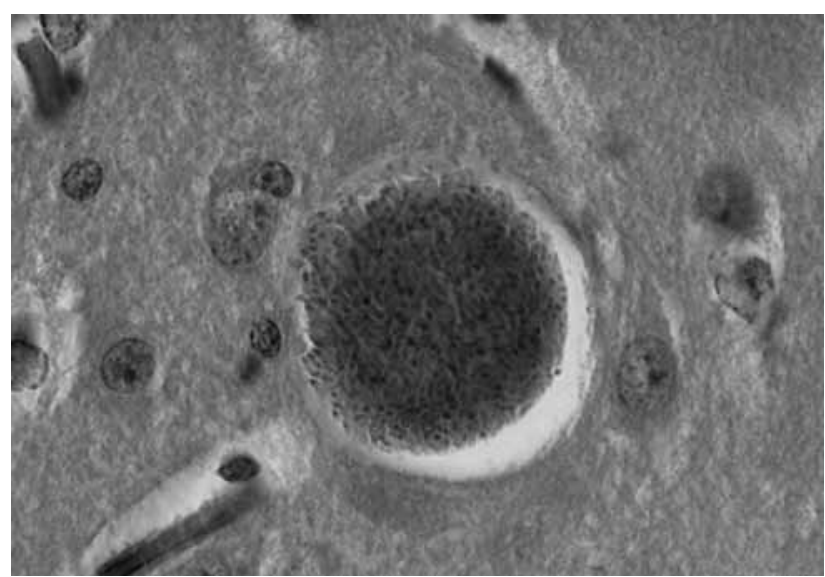

Fig. 2: Photograph showing tissue cyst, neurocytes and red blood cells in rat number $2(\times 400)$

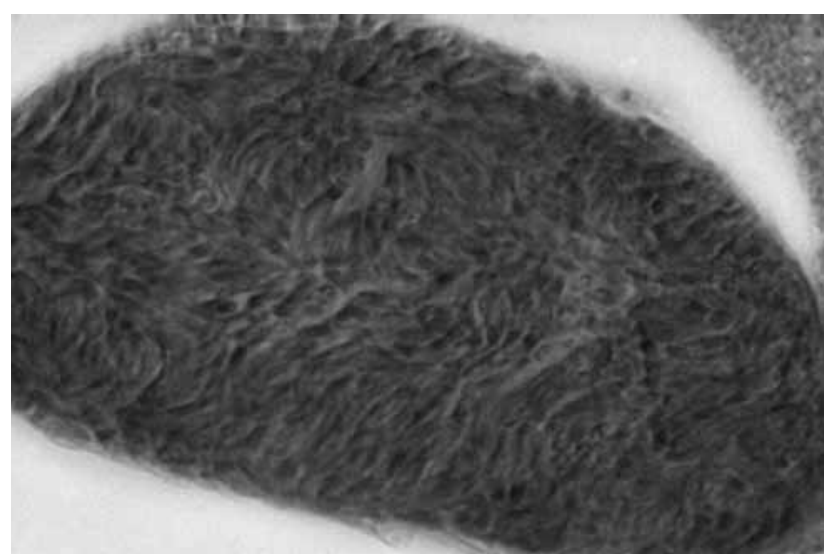

Fig. 3: Photograph showing individual bradyzoites inside tissue cyst in rat number $3(\times 1000)$ 
multiple cysts in the same hemisphere, while the second one had a bilateral infection and the third rat had one single cyst. $T$. gondii cyst was diagnosed according to the morphological appearance. Further molecular study should be performed to confirm the diagnosis.

\section{Discussion}

The local Chow Kit market is the largest wet market in the city of Kuala Lumpur. It is very close to the biggest government hospital in the city centre, Hospital Kuala Lumpur (HKL). This market offers good prices and a wide range of options for visitors. However, the level of cleanliness in this area is always questionable and a matter of concern.

Kuala Lumpur is a rapidly developing city. Modernization and industrialization of this capital city influences the health status, especially with the presence of many legal and illegal foreign workers. In addition, those who cannot cope with the challenges of living in urban areas are plunged into social ills. This description gives an overview of the appropriate circumstances around the Chow Kit market, where prostitution and drug dealers are often reported. According to a study performed in 2003 at HKL, the incidence of toxoplasmic encephalitis in AIDS patients and commercial sex workers was $11 \%$ and $46.6 \%$, respectively (7). Thus, $T$. gondii prevalence around Chow Kit market reflected the rate of infection of $T$. gondii among the local residents.

All water samples in this study were negative for $T$. gondii oocyst. There might be slight water contamination that went undetected because water concentration and microscopic examination was the only method used for detection. Detection of T. gondii oocysts in water is more difficult than that of other coccidian oocysts and there are no standardized methods to perform it. Attempts to recover T. gondii oocysts from water samples in the British Columbia outbreak were unsuccessful (4).

It is known that a polymerase chain reaction (PCR) is one of the most sensitive methods to detect $T$. gondii oocyst in environmental samples and, at present, a bioassay is one definitive way to detect viable oocysts. In fact, both methods are not recommended in our country due to high expenditure and lack of experience. However, in order to determine the presence of $T$. gondii oocyst, we used a sucrose flotation method which was known to be quite sensitive for environmental samples (13).

Rats usually get infected from contaminated soil or by consuming meat containing the infective $T$. gondii cyst. Chicken is a strong possible source of infection in this market. $T$. gondii is proved to be lodged in the heart and brain of hens (2). Hawkers usually slaughtered hens, removed their internal organs, and kept them in open containers which were easily reached by scavenging rats.

We speculate that infection may have changed the habits or behaviors of rats in terms of being less afraid of cats. This may be due to damage of the warning mechanism in the rats through a decreased function of anxiety (14). The amount of stray cats may be one of the reasons for getting a relatively lower number of infected rats in the Chow Kit market. In comparison with the method used in this study, whereby stained histological slides were examined, microscopical examination of squeeze slides from native or homogenized brain tissue is very effective because a bigger volume of brain tissue can be inspected on one slide. The researchers have the intention to use this method in future studies to get more accurate results.

In 2008, the Kuala Lumpur City Hall launched a campaign to encourage hawkers to catch rats on their premises. Free mousetraps have been provided to hawkers, who are paid one Malaysian Ringgit (0.35 USD) for each rat tail. It is hoped that this plan will help to control and prevent the transmission of this parasitic infection. The research group has the intention to do further studies on the isolation of T. gondii tissue cysts from hens, chickens, ducks and repeat the testing of rat and other rodent organs in the market.

The authors declare that they have no competing interest. All authors read and approved the final manuscript. The research was sponsored by the Faculty of Allied Science, Universiti Kebangsaan Malaysia (National Univerisity of Malaysia).

\section{References}

1. Bahia-Oliveira LMG, Jones JL, Azevedo-Silva J, Alves CCF, Oréfice F, Addiss DG. Highly endemic, waterborne toxoplasmosis in north Rio de Janeiro State. Brazil Emerging Infect Dis. 2003; 9:55-62.

2. Dubey JP, Graham DH, Blackston CR, Lehmann T, Gennari SM, Ragozo AMA et al. Biological and genetic characterisation of Toxoplasma gondii isolates from chickens (Gallus domesticus) from São Paulo, Brazil: unexpected findings. Int. J. Parasit. 2004; 1:99-105.

3. Dubey JP, Zarnke R, Thomas NJ, Wong SK, Van Bonn W, Davis JWR et al. Toxoplasma gondii, Neospora caninum, Sarcocystis neurona, and Sarcocystis canis-like infections in marine mammals. Vet. Parasitol. 2003; 116:275-296.

4. Dumètre A. Dardé ML. How to detect Toxoplasma gondii oocysts in environmental samples? FEMS Microbiol. Rev. 2003; 27: 651-661.

5. Lindsay DS, Collins MV, Mitchell SM, Cole R, G. Flick, Wetch CN et al. Sporulation and survival of Toxoplasma gondii oocysts in sea water. J. Eukaryot. Microbiol. 2003; 50:S687-S688.

6. Mohan B, Dubey ML, Malla N, Kumar R. Seroepidemiological study of toxoplasmosis in different sections of population of Union Territory of Chandigarh. Journal of Communicable diseases. 2002; 34(1):15-22.

7. Nissapatorn V, Lee CKC, Khairul AA. Seroprevalence of Toxoplasmosis Among AIDS Patients in Hospital Kuala Lumpur. Singapore Medical Journal. 2003; 4:194-196.

8. Nissapatorn V, Lee CKC, Cho SM. Toxoplasmosis in HIV/AIDS patients in Malaysia. Southeastasian J Trop Med Public Health. 2003; 34:80-5.

9. Shamilah H, Lokman HS, Noor Azain MY, Malkith K, Yusri MY. Seroprevalence of Toxoplasma gondii antibodies in HIV positive and negative patients using the immunofluorescence antibody test (IFAT) methods. Trop Biomed. 2001; 18: $137-41$.

10. Shiba KR, Takeo M, Kazou O et al. High Toxoplasma seroprevalence associated with meat eating habits of locals in Nepal. Asia Pacific Journal of Public Health. 1999; 11(2):89-93.

11. Teutsch SM, Juranek DD, Sulzer A, Dubey JP, Sikes RK. Epidemic toxoplasmosis associated with infected cats. N. Engl. J. Med. 1979; 300:695-699.

12. Veeranoot N. Toxoplasmosis: A scielent threat in Southeast Asia. Research Journal Parasitology. 2007; 2(1):1-12.

13. Villena I, Aubert D, Gomis P, Ferté H, Inglard JC, Denis-Bisiaux H. et al. Evaluation of a strategy for Toxoplasma gondii oocyst detection in water. Appl. Environ. Microbiol. 2004; 70:4035-4039. 
14. Vyas A, Kim SK, Giacomini N, Boothroyd JC, Sapolsky RM. Behavioral Changes Induced By Toxoplasma Infection of Rodents Are Highly Specific to Aversion of Cat Odors. Proceedings of the National Academy of Sciences of the United States of America. 2007; 15:6442-6447.
15. Yue X, Jigang Y, Ning J et al. Seroepidemiology of human Toxoplasma gondii infection in China. BMC Infectious Diseases. 2010; 10:4-7.

16. Zahedi M, Idruss H, Osman S, Salleh F, Ghauth I. Toxoplasmosis in Malaysia: Some epidemiological consideration. J Malaysian Soc Health. 1985; 5:19-23.

\section{Corresponding author:}

Received: $15 / 07 / 2010$

Accepted in revised form: 09/05/2011

Dr. Amal R. Nimir, Department of Pathology, Faculty of Medicine, Cyberjaya University College of Medical Sciences, No. 3410, Jalan Teknokrat 3, Cyber 4, 63000 Cyberjaya, Selangor Darul Ehsan- Malaysia; e-mail address: aralmadi@yahoo.com 